\title{
Junior doctor-led clinical teaching for finals: a useful resource?
}

\author{
Authors: Aiesha Alexander, ${ }^{A}$ Patrick Elder, ${ }^{A}$ Isabelle Svahnström, ${ }^{B}$ Clara Farque $^{B}$ and Oliver Putt ${ }^{B}$
}

\section{Aims}

To investigate the effectiveness of near-peer mock tutorials as a tool to prepare final year medical students for their objective structured long examination record (OSLER) clinical exams.

\section{Methods}

A near-peer revision course was developed for final-year students at a medical school in the West Midlands to aid preparation for their OSLER clinical exams. Junior doctors recruited as volunteer teachers received training in the OSLER assessment method and how to give feedback. Cases were written by the authors to include common clinical scenarios. All final year students $(n=150)$ were invited to participate, with 40 students being enrolled into the program. Weekly mock OSLER tutorials were taught by junior doctors in a classroom setting 3 months leading up to the exams. Pre- and post-teaching questionnaires were administered to students using a 10-point Likert scale format. There were also free-text questions for qualitative feedback. A two-tailed student's t-test, carried out at the $\alpha=0.05$ level, was used to determine statistically significant differences in confidence pre- and post-course.

\section{Results}

Of the 40 attendees, 35 students (87.5\%) completed feedback questionnaires. Responses to pre-course questions ( $n=31$ ) highlighted that $61 \%$ did not feel prepared for the OSLER exam, mainly due to lack of opportunities to practice. Additionally, 10\% of students did not feel confident in their history taking, with confidence also lacking in examination skills (25\%) and answering viva-style questions (33\%). Following the course, the majority of students $(97.1 \%, n=35)$ stated they found the clinical tutorials a helpful way to learn, with $100 \%$ of students stating they felt better prepared for the final year OSLER exam following the teaching sessions. Students reported higher levels of confidence in their history taking (mean 6.67; standard deviation (SD) 1.38 vs mean 7.97; SD 1.54, $t(62)=3.75, p<0.05$ ), examination skills (mean 5.29; SD 1.53 vs 7.31; SD 1.67, $\mathrm{t}(62)=4.99, \mathrm{p}<0.05$ ) and answering viva

Authors: ${ }^{\mathrm{A}}$ University of Warwick, UK; ${ }^{\mathrm{B}}$ University Hospital Coventry and Warwickshire, Coventry, UK style questions (mean 5 ; SD 1.57 vs 7.62; SD 1.64, t(62) =6.46, $\mathrm{p}<0.05)$.

\section{Conclusion}

The benefits of near-peer learning are widely described in medical education research. This study demonstrated that classroombased tutorials, delivered by junior doctors, significantly improved student confidence in preparation for all aspects of their final year OSLER exams. Our results suggest that junior doctor-led teaching is a useful resource for increasing learning opportunities available to students, and offers a valuable and feasible adjunct to faculty teaching.

\section{Conflict of interest statement}

No conflicts of interest to declare. 